\title{
Using the Hidden Markov Model to Improve the Hull-White Model for Short Rate
}

\author{
Nguyet Nguyen, Dung Nguyen, and Thomas P. Wakefield
}

\begin{abstract}
We report a modeling study of short term interest rates using the Hidden Markov Model (HMM) and the Hull-White (HW) model. For this purpose, we modify the original HW model by adding a regime variable to its instantaneous forward function. This variable is defined by the regimes of the short term interest rate which are found by a two-state HMM. The combination of the HMM and the HW model for generating interest rate predictions results in a significant improvement, reducing the error of the estimations by about $50 \%$ compared to that of using HW alone. Furthermore, the errors of the simulations using HMM and HW have a smaller standard deviation compare with which of using the HW. Adjusted R-square results also show that the regime variable is significant. This improvement to the short-term interest rate model has a substantial impact on financial economics and related fields.
\end{abstract}

Index Terms-Interest rate, Hidden Markov Model, simulations, Hull-White, forward rate, regimes.

\section{INTRODUCTION}

The short-term interest rate (short-rate) is an important input for many financial models, especially for financial derivative models, but it is difficult to model accurately. The famous Black-Scholes Model for pricing options is based upon an assumption of a constant short rate. However, in reality, the short rate is not constant, as it fluctuates as economic conditions change.

Classical short rate models, as in [1], [2] assume the short rate follows a Brownian motion process, which, unfortunately, is not supported by empirical evidence. Some of the limitations of these early models were addressed in [3] with the development of the Hull and White Extended Vasicek and Hull and White Extended CIR models. These extensions add more flexibility to the short interest rate models. However, we also experience jumps in the time series of US real interest rates as a result of important macroeconomic changes, most recently in the 2008 economic crisis. Researchers tried to accommodate these jumps by allowing for the possibility of regime-switching in short-term interest rate models. Garcia and Perron [4] modeled the short rate in this manner. These approaches added too much structure to short rate models which most of the time resulted in data over-fitting. Also, the estimations of these models were excessively expensive. The forward rate is the most

Manuscript received January 12, 2018; revised March 10, 2018.

Nguyet Nguyen and Thomas Wakefield are with the Youngstown State University, Youngstown, Ohio, 44555, USA (e-mail: ntnguyen01@ ysu.edu, tpwakefield@ysu.edu).

Dung Nguyen is with the Ned Davis Research Group, Venice, Florida, USA (e-mail dung@ndr.com). important component of the Hull-White model, but has largely been ignored in research. Typically, the forward curve is assumed to be a function of time. However, it is also subject to regime changes due to changes in the macroeconomic environment. Adding dummy regime variables to the forward function is a simple relaxation of the Hull-While model. In this paper, we consider the influence of regimes of the forward rate on the short-term interest rate in the Hull-White model. First, we use the Hidden Markov Model (HMM) to find regimes of the interest rate and code these regimes as dummy variables in the prediction of the forward rate used to generate the short-term interest rate. Using HMM to find regime variables to add to the forward rate function of the Hull-While model is a simple modification but it makes a significant improvement to the HW model. The modification will allow for more accurate short rate modeling, which is critical in the pricing of annuity contracts, investment outcomes and decisions, and in other relevant models in actuarial science and financial mathematics.

The remainder of this paper is organized as follows. Section Two presents an overview of the Hidden Markov Model. Section Three provides a brief introduction of the Hull-White model. The methodology and results of using HMM and HW models to simulate the short term interest are described in Section Four. Section Five concludes the paper.

\section{OVERVIEW OF THE HIDDEN MARKOV MODEL}

The Hidden Markov Model (HMM) was developed by Baum and Petrie in 1966 in [5]. This is a stochastic signal model based upon the following assumptions:

- The observation at time $t$ was generated by some process whose state is hidden.

- The hidden states satisfy the first-order Markov property.

- The transition matrix $A=\left(a_{i j}\right)$ is constant, where $a_{i j}$ is the probability of being in state $S_{j}$ at time $t$ given that the observation at time $t-1$ is in state $S_{i}$.

- The observation at time $t$ of a HMM has a particular probability distribution corresponding to a possible state. The observation probability matrix is denoted by $B$.

The parameters of a HMM are the matrices $A$ and $B$ and the vector $p$. For convenience, we use compact notation for the parameters: $\lambda=\{A, B, p\}$. If the observation probability assumes the Gaussian distribution, then $b_{i}(k)=b_{i}\left(O_{t}=v_{k}\right)=N\left(v_{k}, \mu_{i}, \sigma_{i}\right)$, where $\mu_{i}$ and $\sigma_{i}$ are the mean and variance of the distribution corresponding to the state $S_{i}$, respectively, and $N$ is Gaussian density function. In 
this case, the parameters of HMM are $\lambda=\left\{A, \mu_{i}, \sigma_{i}, p\right\}, i=1,2$.

Since its introduction in 1966, HMM underwent many developments. The innovations primarily involved calibration of the model's parameters. Baum and his colleagues published a maximization method to calibrate HMM's parameters using a single observation in [6]. Sondhi introduced a maximum likelihood estimation method for HMM with multiple observation training, assuming that all the observations are independent in [7]. In 2000, Li, Parizeau, and Plamondon [8] presented a HMM training for multiple observation sequences without the assumption of independence of observations. More details about this model and its algorithms can be found in [9] and [10].

HMM finds applications in many different areas such as speech recognition systems, computational molecular biology, and financial market predictions. Researchers have applied the Hidden Markov Model to forecast stock prices. Hassan and Nath [11] used HMM to predict the stock price for interrelated markets. Kritzman, Page, and Turkington [12] applied HMM with two states to predict regimes in market turbulence, inflation, and industrial production index. Guidolin and Timmermann [13] used HMM with four states and multiple observations to study asset allocation decisions based upon regime switching in asset returns. Ang and Bekaert [14] applied the regime shift model (another name of HMM) for international asset allocation. Nguyen [10] used HMM with both single and multiple observations to forecast economic regimes and stock prices. B. Nobakht, C. E. Joseph and B. Loni [15] implemented HMM by using multiple observation sequences (open, close, low, high) prices of a stock to predict its closing price. Nguyen and Nguyen [16] used HMM for single observation data to predict regimes of some economic indicators and made stock selections based upon the performances of these stocks during the predicted regimes.

Recently, Elliott and Wilson [17] used HMM to model the short-term interest rate by assuming that the mean-reverting level follows a finite-state, continuous-time Markov chain. Erlwein and Mamon [18] implemented HMM for the interest rate model presented by Elliott and Wilson [17] for a financial data set of 30-day Canadian Treasury bill yields. In this paper, we combine HMM with a standard model for the interest rate, the Hull-White model, to generate predictions for the short-term interest rate and compare the results with the results obtained by only using the Hull-White model.

\section{OVERVIEW OF THE HULL-WHITE MODEL}

The short-term interest rate plays a key role in the modeling of financial securities, derivatives, and other interest rate contingent claims. Beginning with Vasicek [2], many models for the short-rate have been proposed. Each model has its advantages and limitations. Two of the earliest models were developed in [1], [2]. Some of the limitations of these early models were addressed by Hull and White [3] with the development of the Hull and White Extended Vasicek and Hull and White Extended CIR models. We provide a brief description of the Vasicek, CIR, and Hull and White models. A detailed description of these and other models for the short-rate can be found in [19].
In the Vasicek model, the risk neutral dynamics are given by the stochastic differential equation

$$
d r(t)=k(\theta-r(t)) d t+\sigma d W(t)
$$

with $r(0)=r_{0}$ and $k, \theta, \sigma, r(0)$ are positive constants. The model is mean-reverting to $\theta$ and implies a normally distributed short-rate. Because the short-rate is normally distributed, the Vasicek model is analytically tractable and produces analytical formulas for bond and option prices. The major drawbacks of the Vasicek model are its inability to match the term structure observed in the market and the possibility of predicting negative rates.

To address and eliminate the possibility of negative rates, Cox, Ingersoll, and Ross [1] proposed a short-rate model of the form

$$
d r(t)=k(\theta-r(t)) d t+\sigma \sqrt{r(t)} d W(t),
$$

with $r(0)=r_{0}>0$ and $k, \theta, \sigma, r(0)$ are positive constants. This CIR model shares the advantage of analytic tractability as the short-rate in this model has a noncentral chi-square distribution. The CIR model also has the advantage of ensuring predicted rates will never be negative. However, it shares the drawback of the Vasicek model that the term structure of the short-term rate can't be matched.

To remedy this issue with the Vasicek and CIR models, Hull and White [3] proposed extensions of both models that are consistent with the current term structure of interest rates. We will examine a version of the Hull-White Extended Vasicek model, as it is the focus of this paper and has the advantage of being analytically tractable. The stochastic differential equation describing the Hull-White Extended Vasicek model is

$$
d r(t)=k(\theta-a(t) r(t)) d t+\sigma(t) d W(t),
$$

where $a(t), \sigma(t)$, and $\theta(t)$ are deterministic functions of time and $\theta(t)$ is chosen to fit the initial term structure of the interest rates and $\sigma(t)$ is to fit the current spot or forward-rate volatility term structure. As noted in [3] and [19], if an exact calibration to the term structure of interest rates is desired, then the perfect fitting of the volatility structure may be problematic. Because of this, we follow the development of a simplified model, analyzed by Hull and White [3], which has the form

$$
d r(t)=(\theta-a r(t)) d t+\sigma d W(t)
$$

where $a$ and $\sigma$ are positive constants and $\theta(t)$ is chosen to exactly fit the current term structure of interest rates observed in the market. We will refer to this as the Hull-White one-factor model. Because this model is an extension of the Vasicek model, it implies a normal distribution for the short rate process $r(t)$ and thus is analytically tractable. However, it has the drawback of the possibility of negative rates.

Le $(\Omega, F, Q)$ be a probability space. Define the market instantaneous forward rate at time 0 for maturity $T$ as

$$
f^{M}(0, T)=-\frac{\partial P^{M}(0, T)}{\partial T} .
$$

Let $E\left(. \mid F_{s}\right.$ and $\operatorname{Var}\left(. \mid F_{S}\right)$ be the expectation and variance conditional on the $F_{s}$ and $\sigma-$ field, respectively. Then it can 
be shown that the Hull-White Model implies a normally distributed short-rate process with

$$
E\left(r(t) \mid F_{s}\right)=r(s) e^{-a(t-s)}+\alpha(t)-\alpha(s) e^{-a(t-s)},
$$

where

and

$$
\alpha(t)=f^{M}(0, T)+\frac{\sigma^{2}}{2 a^{2}}\left[1-e^{-2 a(t-s)}\right]^{2},
$$

$$
\operatorname{Var}\left(r(t) \mid F_{s}\right)=\frac{\sigma^{2}}{2 a}\left[1-e^{-2 a(t-s)}\right]
$$

The Hull-White Model also gives closed formulas for the price of a zero-coupon bond issued at time \$t\$ maturing at time $T, P(t, T)$. The price is given by

$$
P(t, T)=A(t, T) e^{-B(t, T) r(t)}
$$

with

$$
A(t, T)=\frac{P^{M}(0, T)}{P^{M}(0, t)} e^{\left(B(t, T) f^{M}(0, t)-\frac{\sigma^{2}}{4 a}\left(1-e^{-2 a t}\right) B(t, T)^{2}\right)},
$$

and

$$
B(t, T)=\frac{1}{a}\left(1-e^{-a(T-t)} .\right.
$$

\section{Hidden Markov Model AND Hull-White ONE-FACTOR MODEL FOR THE INTEREST RATE}

Because the In this section we explain how to use Hull-White one factor model and Hidden Markov Model to simulate the interest rate. We first present the methodology, followed by the data preparation and numerical results.

\section{A. A. Methodology}

Consider the time interval $t_{0}, t_{1}, \ldots, t_{N}$ with equal width $\Delta_{t}=\frac{1}{N}$. The short interest rate series $r_{t_{0}}, r_{t_{1}}, \ldots, r_{t_{N}}$ can be simulated recursively by using the Hull-White one factor model in equation (1) with an initial interest rate $r_{0}$ :

$$
r_{t_{i}}=\theta\left(t_{i-1}\right) \Delta_{t}+\left(1-\mathrm{a} \Delta_{\mathrm{t}}\right) \mathrm{r}_{\mathrm{t}_{\mathrm{i}-1}}+\sigma \sqrt{\Delta_{\mathrm{t}}} \mathrm{Z}_{\mathrm{t}_{\mathrm{i}}},
$$

where the deterministic function $\theta(t)$ is chosen as

$$
\theta(t)=\frac{\partial}{\partial t} f(0, t)+a f(0, t)+\frac{\sigma^{2}}{2 a}\left(1-e^{-2 a t}\right) .
$$

Hull and White [3] showed that we can eliminate the term $\frac{\sigma^{2}}{2 a}\left(1-e^{-2 a t}\right)$ in Equation (3) since its value is fairly small. Therefore we just use

$$
\theta(t)=\frac{\partial}{\partial t} f(0, t)+a f(0, t)
$$

In the Hull-White model, the market instantaneous forward function $f(0, t)$, the forward rate at time 0 for maturity $t$, was assumed to be a third degree polynomial function of time $t$ of the form

$$
f(0, t)=b_{0}+b_{1} t+b_{2} t^{2}+b_{3} t^{3} .
$$

In the HMM-HW model, we add a state variable for the interest rate, denoted $\lambda$, into the instantaneous forward function defined as a dummy variable. Thus the forward function has the form

$$
f(0, t)=c \lambda+b_{0}+b_{1} t+b_{2} t^{2}+b_{3} t^{3} .
$$

First, we use HMM to find the states of the interest rate, the dummy variable $\lambda$ of the forward rate function, then we use the polynomial regression model to find coefficients of the forward function in Equation (6). Using equations (4) and (7) gives

$$
\begin{gathered}
r_{t_{i}}-r_{t_{i-1}}=\frac{\partial}{\partial t} f\left(0, t_{i-1}\right) \Delta_{t}+a\left(f\left(0, t_{i-1}\right)-r_{t_{i-1}}\right) \Delta_{t}+ \\
\sigma \sqrt{\Delta_{t}} Z_{t_{i}}
\end{gathered}
$$

Thus

$$
\begin{gathered}
\sigma \sqrt{\Delta_{t}} Z_{t_{i}}=r_{t_{i}}-r_{t_{i-1}}-\frac{\partial}{\partial t} f\left(0, t_{i-1}\right) \Delta_{t}+a\left(f\left(0, t_{i-1}\right)-\right. \\
r t i-1 \Delta t
\end{gathered}
$$

We then find $a$ as a minimizer of function

$$
\begin{aligned}
& H W(a)=\sum_{i=1}^{N}\left(r_{t_{i}}-r_{t_{i-1}}-\frac{\partial}{\partial t} f\left(0, t_{i-1}\right) \Delta_{t}+\right. \\
& \text { afo,ti-1-rti-1 } 1 \text { t) } 2 \text {. }
\end{aligned}
$$

The last parameter that we need to calibrate is $\sigma$. To find $\sigma$, we first predict the interest rate $\hat{r}$ using the formula

$$
\begin{gathered}
\hat{r}_{i}=\left(\frac{\partial}{\partial t} f\left(0, t_{i-1}\right)+a f\left(0, t_{i}\right)\right) \Delta_{t}+\left(1-a \Delta_{t}\right) r_{t_{i-1}}, \\
\text { for } i=1,2, \ldots, N \text {, and } \hat{r}_{t_{0}}=r_{t_{0}} \text {. Then } \\
\sigma=\frac{s}{\sqrt{\Delta_{t}}},
\end{gathered}
$$

where $s$ is the standard deviation of the error series $\hat{r}_{t_{i}}$ $r_{t_{i}}, i=1, \ldots, N$.

After calibrating all the needed parameters, we then simulate the interest rate using Equation $\sim(\operatorname{|ref}\{$ Rate $\})$. Data and results of the simulations will be presented in the next section.

\section{B. Implementation and Results}

We use daily data on the one-year interest rate in this study. The economic crisis in 2008 had huge effects on many economic indicators, including the interest rate. Therefore we want to use HMM to detect the crisis time and find regimes of the interest rate. We use HMM with two regimes, which represent the two main economic conditions: growth and recession. We assume that the observed probability distributions corresponding to these two regimes are Gaussian in nature. Daily data of the one-year interest rate from December 31, 2004, to January 1, 2010, is used to calibrate parameters and find the data's regimes, using Baum-Welch and Viterbi algorithms. First, we use the Baum-Welch algorithm to calibrate the model parameters $\lambda=\left\{A, \mu_{i}, \sigma_{i}, p\right\}, i=1,2$. Then we calculate the ratio of the means and variances of the two normal distributions, $\frac{\mu_{i}}{\sigma_{i}}$, and define regime one as the regime of the normal distribution with a higher ratio.

The trained transition probability matrix is

$$
\mathrm{A}=\left[\begin{array}{ll}
1.0000 & 0.0000 \\
0.0013 & 0.9987
\end{array}\right]
$$


The initial probability of being in regime one or two at time 0 is $p=(0,1)$.

The parameters of the two normal distributions corresponding to the two regimes are: $\mu_{1}=4.34, \sigma_{1}=0.73$, and $\mu_{2}=0.84, \sigma_{2}=0.0 .72$. The regimes of the short term interest rate from December 31, 2004, to January 1, 2010 are presented in Fig. 1. The result shows that the interest rate in the period had two regime periods: Regime One from December 31, 2004, to December 31, 2007, and Regime Two from January 1, 2008, to January 1, 2010. We pick a period of ten months before the regime change on December 31, 2007 and ten months after the regime change to make a pool of data for the interest rate simulations. We choose to expand the data to ten months before and after the regime change date because we want a pool of data that has only two time periods consisting of the first regime and the second regime. The transition date between these two regimes is December 31, 2007. The number of points in the data pool is the number of business days during the period, which is 450 days. Each year has 252 working days.

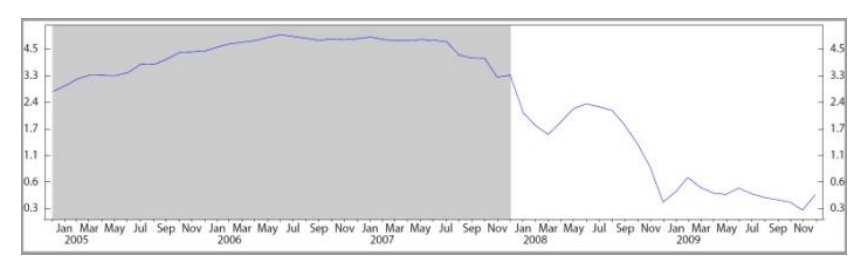

Fig. 1. Predicted regimes of one-year interest rate using HMM.

Therefore, we choose randomly one time series of 252 consecutive points from the pool of data to train parameters for the Hull-White model and use the trained parameters to simulate the interest rate. Thus, the time step in the HW model is $\Delta_{t}=\frac{1}{252}$. Since we used HMM to find regimes of the data pool, for any random one-year-period series in the data pool, we already know its regimes which were switched on December 31, 2007. We follow the methodology presented in the previous section to simulate the one-year interest rate using a random daily time series from the pool starting on October 30, 2007 to October 28, 2008. The regime switch date corresponds to the time variable $t=42 / 252$.

First, based on its regimes, found by using HMM, we determine the values of a regime (or dummy) variable $\lambda_{t}$ in Equation (6). Here

$$
\lambda_{t}=\left\{\begin{array}{l}
1 \text { if } 0 \leq t \leq \frac{42}{252} \\
0 \text { if } \frac{42}{252}<t \leq 1
\end{array}\right.
$$

We then use polynomial regression to determine the instantaneous forward rate function represented by Equation (6):

$$
\begin{aligned}
& f(0, t)=-0.8925 \lambda_{t}+4.0214-9.1573 t+20.6133 t^{2}- \\
& 13.3839 t^{3} .
\end{aligned}
$$

Thus, the derivative of $f(0, t)$ with respect to $t$ is:

$$
f(0, t)=-9.1573+41.2266 t-40.1517 t^{2} .
$$

We substitute these functions into the function $H W(a)$ in Equation (9) and use least squares regression to optimize the function and find its minimizer $a$. Then we predict the sequence $\hat{r}$ using Equation (10) and calibrate the parameter $\sigma$ using Equation (11). Finally, we compute the function $\theta(t)$ using Equation (4).

We have the parameters $a=27.5136, \sigma=1.4797 \$$, and

$\theta(t)=-9.1573+41.2266 t-40.1517 t^{2}+$ $27.5136\left(-0.8925 \lambda_{t}+4.0214-9.1573 t+20.6133 t^{2}-\right.$ $\left.13.3839 t^{3}\right)$.

After determining all of HW's parameters $(a, \sigma, \theta)$, we use the parameters to simulate the interest rate for the same period using Equation (7). The results of the simulation are presented in Fig. 2. In Fig. 2, we plot the three series: real interest rate, the simulated interest rate using the original HW model, and the simulated interest rate using HMM-HW model.

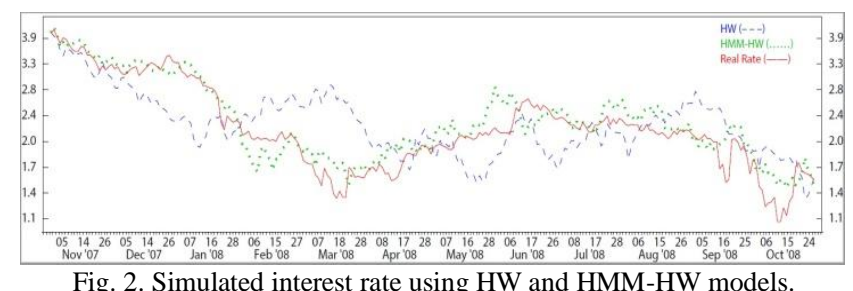

Fig. 2. Simulated interest rate using HW and HMM-HW models.

We compute errors of simulations using the absolute percentage error (APE), the average absolute error (AAE), the average relative percentage error (ARPE) and the root-mean-square error (RMSE). These error estimators are calculated using the formulas

$$
\begin{aligned}
A P E & =\frac{1}{\bar{r}} \sum_{i=1}^{N} \frac{\left|r_{i}-\tilde{r}_{i}\right|}{N} \\
A A E & =\sum_{i=1}^{N} \frac{\left|r_{i}-\tilde{r}_{i}\right|}{N} \\
A R P E & =\frac{1}{N} \sum_{i=1}^{N} \frac{\left|r_{i}-\tilde{r}_{i}\right|}{N} \\
R M S E & =\sqrt{\frac{1}{N} \sum_{i=1}^{N} \frac{\left|r_{i}-\tilde{r}_{i}\right|}{N}}
\end{aligned}
$$

where $N$ is a number of simulated points, $r_{i}$ is the real interest rate, $\tilde{r}_{i}$ is the simulated rate, and $\bar{r}_{i}$ is the mean of the sample. The errors are listed in Table I. The results in Table I show that the HMM-HW reduces errors of the interest simulations by about $50 \%$.

We repeat the process for 1500 random series in the pool and plot the histogram of the errors, calculated by formulas (12)-(15), in Figs. 3-6, respectively. The results show that the HMM-HW model reduces not only the mean of the errors but also their variance. We see from the figures that the histogram of HMM-HW's errors is shifted to the left compared with the histogram of HW's errors. Furthermore, the distributions of errors of these two methods for the interest rate have positive skewness and their kurtosis is far from the kurtosis of the normal distribution.

We also test the efficiency of adding the regime (dummy) variable to HW by calculating the adjusted $R^{2}$ of regressions and plot the results in Fig. 7.

In Fig. 7, the horizontal axis is the end date of each series of these 1500 simulations, and the vertical axis is the adjusted $R^{2}$. The adjusted $R^{2}$ was calculated by using the formula

$$
R_{a d j}=1-\frac{\left(1-R^{2}\right)(N-1)}{N-k-1}
$$


where $N$ is sample size and $k$ is the number of variables in the model, excluding the constant. If a useless variable is added to a regression model, the adjusted $R^{2}$ will decrease. In contrast, if we add a useful variable to the model, the adjusted $R^{2}$ will increase.The results show that the regime variable is a significant variable in the forward function. The adjusted $R^{2}$ increased when we added the dummy variable to the model.

TABLE I. ERRORS OF SIMULATING INTEREST RATE USING HW AND HMM-HW MODELS

\begin{tabular}{lllcc}
\hline \hline Models & AAE & RMSE & ARPE & APE \\
\hline HW & 0.4617 & 0.5899 & 0,0018 & 0,2034 \\
HMM-HW & 0.2150 & 0.2778 & 0.0009 & 0.0947 \\
\hline \hline
\end{tabular}

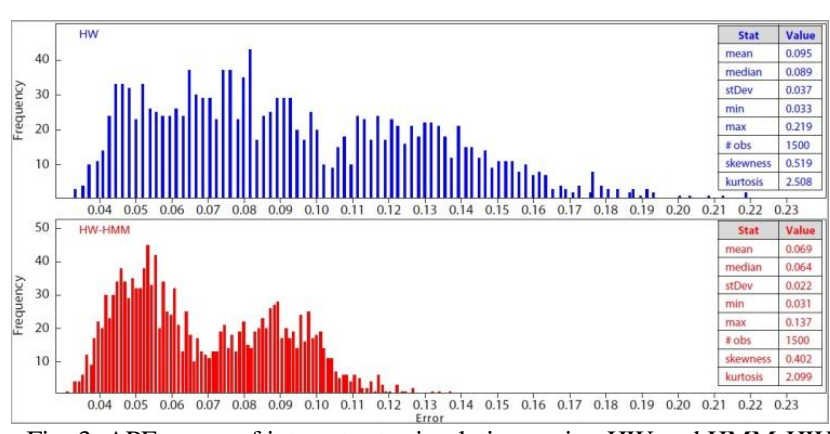

Fig. 3. APE errors of interest rate simulations using HW and HMM-HW models.

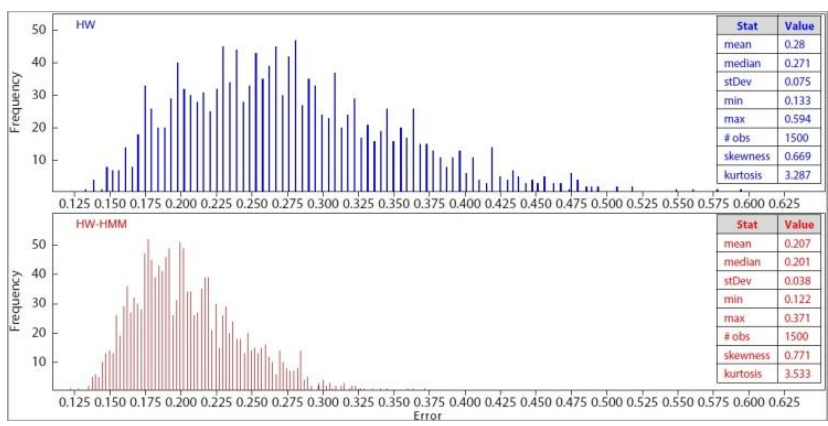

Fig. 4. AAE errors of interest rate simulations using HW and HMM-HW models.

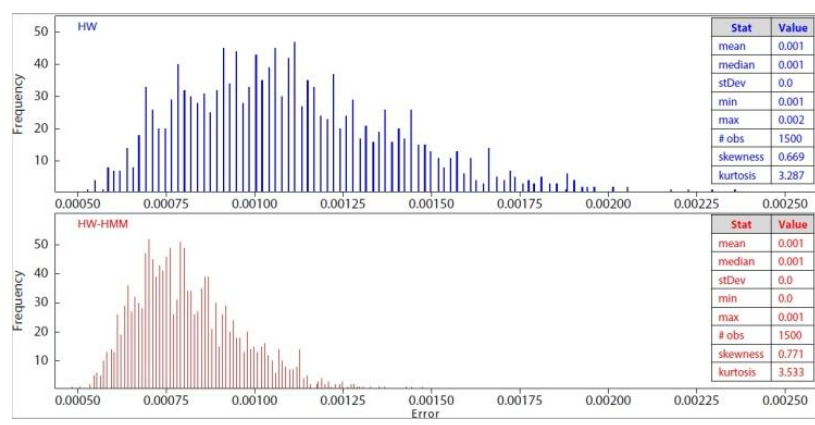

Fig. 5. ARPE errors of interest rate simulations using HW and HMM-HW models.

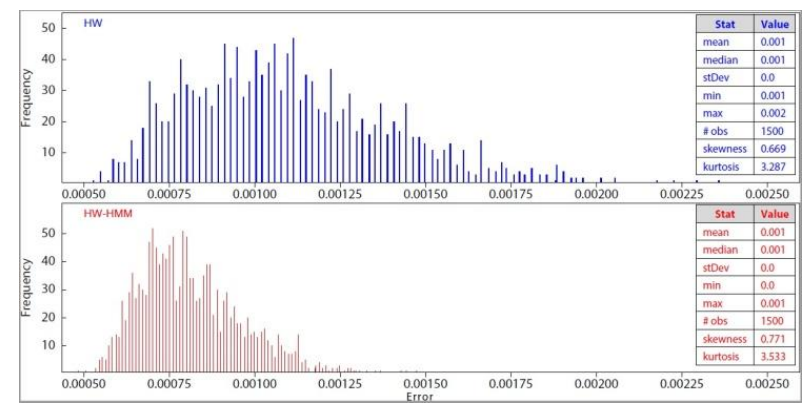

Fig. 6. RMSE errors of interest rate simulations using HW and HMM-HW models.

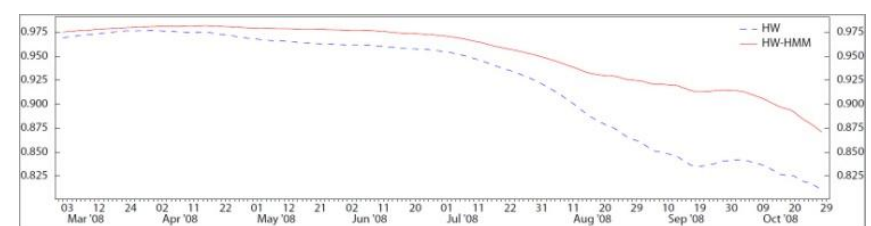

Fig. 7. Adjusted $R^{2}$ of regression of forward function in HW and HMM-HW models.

\section{CONCLUSION}

The Hull-White one-factor model is a classical model for the short-term interest rate. The forward rate function in the HW model is assumed to be a function of time $t$. However, we found jumps in the time series of U.S. real interest rates as a result of important macroeconomic changes, most recently during the 2008 economic crisis. Therefore, we investigated the effects of the jumps on short term interest rate modeling.

In this study, we added a regime variable to the forward rate function to make it more adapt to the regime switch of the interest rate. The regimes were identified by the two-state (or two-regime) HMM. Adding the dummy regime variable to the forward function is a simple relaxation of the Hull-White model. The modification made a significant improvement to the HW model. The error of the interest rate estimations using HMM and HW (HMM-HW) model was reduced by half compared with the original HW model. Furthermore, the results of the short term interest rate simulations showed that the HMM-HW's histogram errors were shifted to the left and shrunk horizontally by half of the size of the HW's histogram errors. Hence using the HMM-HW model for simulating short the term interest rates reduced not only the mean but also the variance of errors. Additionally, the adjusted $R^{2}$ of the regression model for the forward rate function was increased when we added the regime variable. All of these results indicate that the regime variable has a significant influence in modeling the interest rate. This important improvement of the short-term interest rate model has a substantial impact on the pricing of annuity contracts, investment outcomes and decisions, and in other relevant models in actuarial science and financial mathematics.

\section{REFERENCES}

[1] J. C. Cox, J. E. Ingersoll, and S. A. Ross, "A theory of the term structure of interest rates," Econometrica, vol. 53, pp. 363-384, 1985.

[2] O. A. Vasicek, "An equilibrium characterization of the term structure," Journal of Financial Economics, vol. 5, pp. 177-188, 1977.

[3] J. Hull and A. White, "Pricing interest-rate-derivative securities," The Review of Financial Studies, vol. 3, pp. 573-592, 1990.

[4] G. Rene and P. Perron, "An analysis of the real interest rate under regime shifts," The Review of Economics and Statistics, vol. 78, no. 1, pp. 111-125, 1996.

[5] L. E. Baum and T. Petrie, "Statistical inference for probabilistic functions of finite state Markov chains," The Annals of Mathematical Statistics, vol. 37, pp. 1554-1563, 1966.

[6] L. E. Baum, T. Petrie, G. Soules, and N. Weiss, "A maximization technique occurring in the statistical analysis of probabilistic functions of Markov chains," The Annals of Mathematical Statistics, vol. 41, no. 1, pp. 164-171, 1970.

[7] S. E. Levinson, L. R. Rabiner, and M. M. Sondhi, "An introduction to the application of the theory of probabilistic functions of Markov process to automatic speech recognition," Bell System Technical Journal, vol. 62, no. 4, pp. 1035-1074, 1983.

[8] X. Li, M. Parizeau, and R. Plamondon, "Training Hidden Markov Models with multiple observations - A combinatorial method," IEEE Transactions on PAMI, vol. 22, no. 4, pp. 371-377, 2000. 
[9] L. R. Rabiner, "A tutorial on hidden Markov models and selected applications in speech recognition," IEEE, vol. 77, pp. 257-286, 1989.

[10] N. Nguyen, "Probabilistic methods in estimation and prediction of financial models," Ph.D. dissertation, University of Florida, 2014.

[11] M. Hassan and B. Nath, "Stock market forecasting using hidden Markov models: A new approach," in Proc. IEEE Fifth International Conference on Intelligent Systems Design and Applications, 2005.

[12] M. Kritzman, S. Page, and D. Turkington, "Regime shifts: Implications for dynamic strategies," Financial Analysts Journal, vol. 68, no. 3, pp. 22-39, 2012.

[13] M. Guidolin and A. Timmermann, "Asset allocation under multivariate regime switching," FRB of St. Louis Working Paper No. 2005-002C, 2006.

[14] A. Ang and G. Bekaert, "International asset allocation with regime shifts," The Review of Financial Studies, vol. 15, no. 4, pp. 1137-1187, 2002.

[15] B. Nobakht, C. E. Joseph, and B. Loni, "Stock market analysis and prediction using Hidden Markov Models," in Proc. the Student Conference on Engineering and Systems (SCES), 2012, pp. 1-4.

[16] N. Nguyen and D. Nguyen, "Hidden Markov Model for stock selection," Risks, vol. 3, no. 4, pp. 455-473, 2015.

[17] J. R. Elliott and C. A. Wilson, The Term Structure of Interest Rates in a Hidden Markov Setting, Berlin: Springer, 1995.

[18] C. Erlwein and R. Mamon, An Online Estimation Scheme for a Hull-White Model with HMM-Driven Parameters, Berlin: Springer, 2007.

[19] D. Brigo and F. Mercurio, Interest Rate Models: Theory and Practice, Berlin: Springer, 2006.

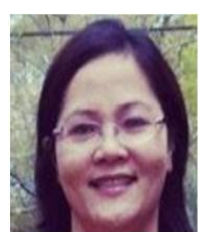

Nguyet Nguyen was born in Vietnam. She received her bachelor of mathematical education and master of mathematical science from Hanoi, National University of Education, Vietnam in 1998 and 2002 respectively. She got her master in 2011 and $\mathrm{PhD}$ in 201 in financial mathematics from Florida State University.

Nguyen is currently an assistant professor in mathematics and statistics department at Youngstown State University, $\mathrm{OH}$ USA. Her research interests and activities are focused on computational finance, actuarial science, and the Monte Carlo method.

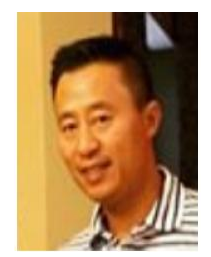

Dung Nguyen received his bachelor's degree from Hanoi National Economics University, Dung Nguyen and completed master's degrees in economics, financial mathematics, and international development. Also, he is receiving his $\mathrm{PhD}$ in economics from Florida State University, USA.

Dung works as a senior quantitative analyst at Ned Davis Research Group, Venice, Florida, USA. Hi research interests include applying dynamic general equilibrium models on the labor market, social security benefits and the aging population.

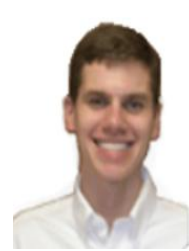

Thomas Wakefield completed a $\mathrm{PhD}$ in pure mathematics at Kent State University in 2008 and recently became a fellow of the Society of Actuaries in August 2015.

Thomas is a professor in mathematics and statistics at Youngstown State University. He has 13 publications, 12 in algebra and one in actuarial science. He received an $\mathrm{AB}$ in economics from Youngstown State and is also conducting research in operations research and supply chains. 